\title{
STRUCTURE AND INPUT-OUTPUT PROPERTIES IN NETWORKS OF NONLINEAR SYSTEMS
}

Murat Arcak

REGENTS OF THE UNIVERSITY OF CALIFORNIA THE

$08 / 26 / 2014$

Final Report

DISTRIBUTION A: Distribution approved for public release.

Air Force Research Laboratory AF Office Of Scientific Research (AFOSR)/ RTA

Arlington, Virginia 22203

Air Force Materiel Command 


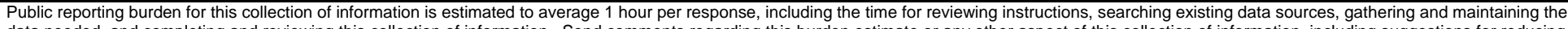

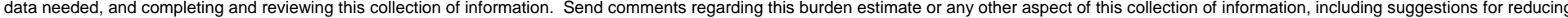

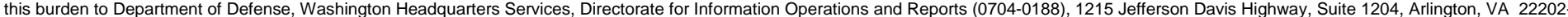

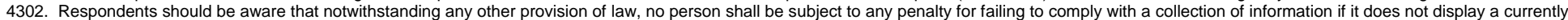
valid OMB control number. PLEASE DO NOT RETURN YOUR FORM TO THE ABOVE ADDRESS.

\begin{tabular}{l|l} 
1. REPORT DATE (DD-MM- $Y Y Y Y)$ & 2. REPORT TYPE
\end{tabular} 20-08-2014 Final

4. TITLE AND SUBTITLE

Structure and Input-Output Properties in Networks of Nonlinear Systems

COVERED (FrOM - To

15-08-2011 to 14-08-2014

5a. CONTRACT NUMBER

5b. GRANT NUMBER

FA9550-11-1-0244

5c. PROGRAM ELEMENT NUMBER

6. AUTHOR(S)

Murat Arcak

5d. PROJECT NUMBER

5e. TASK NUMBER

5f. WORK UNIT NUMBER

7. PERFORMING ORGANIZATION NAME(S) AND ADDRESS(ES)

8. PERFORMING ORGANIZATION REPORT

University of California Berkeley NUMBER

Sponsored Projects Office

Berkeley, CA. 94704-5940

9. SPONSORING I MONITORING AGENCY NAME(S) AND ADDRESS(ES)

10. SPONSOR/MONITOR'S ACRONYM(S)

DAF Air Force Office of Scientific Research

11. SPONSOR/MONITOR'S REPORT NUMBER(S)

\section{DISTRIBUTION I AVAILABILITY STATEMENT}

Approved for public release

\section{SUPPLEMENTARY NOTES}

\section{ABSTRACT}

In this project we developed an input-output approach to predict and engineer collective behavior in networks. This approach overcomes the complexity of the large-scale, nonlinear dynamical model by dividing the analysis and design tasks into two layers: At the network layer, we represent the nodes with appropriate input-output properties as abstractions of their detailed models and exploit these properties in conjunction with the interconnection structure to ascertain desirable behaviors. At the network layer, we represent the nodes with appropriate input-output properties as abstractions of their detailed models and exploit these properties in conjunction with the interconnection structure to ascertain desirable behaviors. At the node layer, we study the individual dynamical models to verify or assign the input-output properties without relying on knowledge of global network properties.

\section{SUBJECT TERMS}

dynamical systems, control, networks

\begin{tabular}{|c|c|c|c|c|c|}
\hline \multicolumn{3}{|c|}{ 16. SECURITY CLASSIFICATION OF: } & \multirow{2}{*}{$\begin{array}{l}\text { 17. LIMITATION } \\
\text { OF ABSTRACT }\end{array}$} & \multirow{2}{*}{$\begin{array}{l}\text { 18. NUMBER } \\
\text { OF PAGES } \\
6\end{array}$} & $\begin{array}{l}\text { 19a. NAME OF RESPONSIBLE PERSON } \\
\text { Murat Arcak }\end{array}$ \\
\hline a. REPORT & b. ABSTRACT & c. THIS PAGE & & & $\begin{array}{l}\text { 19b. TELEPHONE NUMBER (include area } \\
\text { code) } \\
510-642-4804\end{array}$ \\
\hline
\end{tabular}




\title{
Structure and Input-Output Properties in Networks of Nonlinear Systems - Final Report
}

\author{
Murat Arcak \\ Principal Investigator \\ Department of Electrical Engineering and Computer Sciences \\ University of California, Berkeley \\ AFOSR Dynamical Systems and Control Department \\ Dynamics and Control Program \\ FA9550-11-1-0244
}

\begin{abstract}
Networks of interacting components permeate science and engineering. The common paradigm is the ability of lower-level components to generate higher-level sophistication without centralized intelligence. Increased understanding of the interplay between network structure and the resulting dynamics is now enabling researchers to design emergent behaviors for engineering applications, such as cooperative robotics and synthetic biology.

In this project we developed an input-output approach to predict and engineer collective behavior in networks. This approach overcomes the complexity of the large-scale, nonlinear dynamical model by dividing the analysis and design tasks into two layers: At the network layer, we represent the nodes with appropriate input-output properties as abstractions of their detailed models and exploit these properties in conjunction with the interconnection structure to ascertain desirable behaviors. At the node layer, we verify or assign the input-output properties without relying on knowledge of global network properties.

Automated methods for verification of complex, adaptive, nonlinear systems is a key enabler for the Air Force mission. The analysis and design tools resulting from this project were applied to multi-agent systems, biological networks, and to traffic networks. Conceptual unification of ideas from diverse types of networks is of fundamental importance for network-centric operations which call for core concepts and widely applicable tools.
\end{abstract}

\section{Results}

\subsection{Stability Certification for Networks from Input-Output Properties}

This project developed an input-output approach to networks that is uniquely capable of overcoming the complexity of large-scale dynamical models. This approach abstracts input-output properties of the components, rather than dealing with detailed dynamical models, and exploits the coupling structure to predict and engineer collective behavior. 
One of our key contributions was to exhibit special interconnection structures amenable to this approach in diverse types of networks. In [1], we applied this approach to cooperative control problems and demonstrated its design flexibility. Unlike the point mass models commonly used in the literature, we use a passivity property inherent in rigid body models for satellites, robots, marine vehicles, etc. The research monograph [1], published by Springer, presents new robust and adaptive designs demonstrating the power and flexibility of the passivity approach in cooperative control design. We further extended these results to solve the problem of decentralized attitude synchronization of rigid bodies [A7], and to design distributed control strategies for a team of mobile agents that allows for size-scaling of a formation while maintaining the formation shape [A8]. We have solved this problem without relying on direct communication between the agents, using only relative distance information to propagate cues about the desired size from a leader.

We then addressed several practically important problems to expand the applicability of the input-output approach: Because the network equilibrium depends on the interconnection, we proposed a notion of equilibrium-independent dissipativity and provided analytical and numerical tools for its verification [A3]. With this notion, the components of the network are now modular and stability is established without explicit knowledge of the network equilibrium. This result is particularly important for biochemical reaction networks where the steady-state depends on highly uncertain system parameters. Using equilibrium-independent passivity and graph-theoretic concepts, we developed a stability criterion for a class of reaction networks that exhibit intertwined feedback loops as in metabolic networks and oscillator circuits [A4].

Stochastic models, which are particularly important for biochemical reactions, are studied with new stochastic passivity notions in [A9]. In [A10], we defined a "frequency roll-off" property that captures time-scale information about the components and combined this property with passivity to address time delays in the network links. This result provides a means to smoothly interpolate among stability conditions provided by passivity analysis and the small-gain theorem, as the bound on the time delay varies from zero to infinity.

\subsection{Synchronization and Spatial Pattern Formation}

In collaboration with colleagues in synthetic biology, we started investigating how critical processes in natural multicellular organisms can be reestablished in a bacterial medium to enable synthetic multicellular systems. A problem of particular interest is spatial gene expression patterns that allow distinct cell types to emerge in the early development of natural organisms.

Using our analysis and design tools described above, we proposed a novel gene network that spontaneously generates such patterns with the help of a diffusible molecule establishing cell-to-cell communication [A5]. The architecture of this network is grounded in feedback control principles and the resulting pattern formation is interpreted as a nonminimum phase phenomenon in [A23]. This network incorporates an oscillator circuit combined with a feedback loop that quenches the oscillations and contains a diffusible molecule. As demonstrated mathematically in [A5], diffusion of this molecule allows spatial modes with high wave numbers to grow into a spatio-temporal pattern. The proposed architecture is amenable to synthetic implementation since oscillator circuits already exist. The publication [A5] has been received well by the synthetic biology community, and we are 
now implementing this gene network in vivo [A21].

While investigating the mathematical principles of diffusion-driven pattern formation, we also developed new insights about the converse problem of synchronization. Synchronization is essential for several physiological processes, such as circadian rhythms, insulin secretion, cardiac function, and for engineering systems, such as multi-machine power systems and multi-agent systems. In [A2] and [A12], we studied reaction-diffusion PDE models for spatially distributed processes and presented analytical and numerical tests that guarantee spatially homogeneous solutions. As demonstrated in these references, the new tests provide dramatic improvements over existing spatial homogeneity criteria in the mathematical biology literature.

By abstracting the coupling structure generated by diffusion, we next addressed a broad class of networks where the feedback to each node is a local sum of differentials with respect to neighbors. This form of coupling arises in coordination of multi-agent systems, electromechanical coupling of synchronous machines, and macroscopic models of reaction-diffusion systems. In [A6], we studied networks with this coupling structure and asked how the coupling weights should be assigned to enhance synchronization properties. We then developed convex optimization algorithms that solve this problem with the help of tools from spectral graph theory.

Our synchronization criteria rely on sufficient connectivity, as measured by the second smallest eigenvalue $\lambda_{2}$ of the Laplacian matrix describing the interconnection graph. Suppose now that the connectivity is initially too weak to afford synchrony. Can the the link weights be updated locally to achieve synchronization? We addressed this problem in [A15] and developed an adaptive scheme that increases the weight links according to the synchronization error between the nodes connected by each link.

In a separate line of work, we investigated pattern formation enabled by cell-to-cell contact rather than by diffusion. We developed a broadly applicable dynamical model for cellular interactions and revealed the key system properties that enable spatial patterns to emerge [A11]. We then used graph partitioning concepts to predict what type of patterns emerge for each type of interconnection graph [A13].

\subsection{Further Results and Conclusions}

Encouraged by the results summarized above, we started broadening the scope of our research on networked systems. In [A17], we extended our input-output approach to "safety" verification where the goal is to ensure that no trajectory enters an undesirable region in the state-space. We derived convex programs to search for a weighted sum of storage functions, each associated with an input-output property, such that a sublevel set excluding the unsafe set exists. A related task is to design switching rules between the discrete modes of a hybrid system to achieve a safety specification that cannot be met within a single mode. We presented preliminary results for this task in [A22] and applied them to multi-agent surveillance problems. We further started investigating the applicability of our design and analysis tools to traffic networks. Preliminary results in this direction are presented in [A14] and [A16].

In summary, major contributions were made in this project to enable efficient analysis and synthesis of networked dynamical systems. The results were applied to multi-agent systems, biological 
networks, and to traffic networks. Conceptual unification of ideas from diverse types of networks is of fundamental importance for network-centric operations which call for core concepts and widely applicable tools. The Dynamics and Control Program at the Air Force Office of Scientific Research has been instrumental in enabling major advances in the field, and we greatly appreciate the support received for this project.

\section{Publications under this Award}

\section{Book:}

[A1] He Bai, Murat Arcak and John Wen. Cooperative Control Design: A Systematic, Passivity-Based Approach. Springer, Communications and Control Engineering Series, 2011.

\section{Book Chapter:}

[A2] Zahra Aminzare, S. Yusef Shafi, Murat Arcak and Eduardo Sontag. Guaranteeing spatial uniformity in reaction-diffusion systems using weighted $\mathcal{L}_{2}$-norm contractions. In A Systems Theoretic Approach to Systems and Synthetic Biology: Models and System Characterizations, V. V. Kulkarni, G.-B. Stan and K. Raman, editors, volume 1, chapter 3, Springer Verlag, 2014.

\section{Journal Papers:}

[A3] George Hines, Murat Arcak, Andrew Packard. Equilibrium-independent passivity: A new definition and numerical certification. Automatica, vol.47, no.9, pages 1949-1956, 2011.

[A4] Murat Arcak. Diagonal stability on cactus graphs and application to network stability analysis. IEEE Transactions on Automatic Control, vol.56, no.12, pages 2766-2777, 2011.

[A5] Justin Hsia, William J. Holtz, Daniel C. Huang, Murat Arcak, Michel M. Maharbiz. A feedback quenched oscillator produces Turing patterning with one diffuser. PLoS Computational Biology, vol.8, no.1, pages e1002331, 2012.

[A6] S. Yusef Shafi, Murat Arcak, Laurent El Ghaoui. Graph weight allocation to meet Laplacian spectral constraints. IEEE Transactions on Automatic Control, vol.57, no.7, pages 1872-1877, 2012.

[A7] Christopher G. Mayhew, Ricardo G. Sanfelice, Jansen Sheng, Murat Arcak, Andrew R. Teel. Quaternion-based hybrid feedback for robust global attitude synchronization. IEEE Transactions on Automatic Control, vol.57, no.8, pages 2122-2127, 2012.

[A8] Samuel Coogan and Murat Arcak. Scaling the size of a formation using relative position feedback. Automatica, vol.48, no.10, pages 2677-2685, 2012. 
[A9] Ana S. Rufino Ferreira, Murat Arcak, and Eduardo Sontag. Stability certification of large scale stochastic systems using dissipativity. Automatica, vol.48, no.11, pages 29562964, 2012.

[A10] Erin Summers, Murat Arcak, Andrew Packard. Delay robustness of interconnected passive systems: An integral quadratic constraint approach. IEEE Transactions on Automatic Control, vol.58, no.3, pages 712-724, 2013.

[A11] Murat Arcak. Pattern formation by lateral inhibition in large-scale networks of cells. IEEE Transactions on Automatic Control, vol.58, no.5, pages 1250-1262, 2013.

[A12] Yusef Shafi, Murat Arcak, Mihailo Jovanović and Andrew Packard. Synchronization of diffusively-coupled limit cycle oscillators. Automatica, vol.49, no.12, pages 3613-3622, 2013.

[A13] Ana S. Rufino Ferreira and Murat Arcak. A graph partitioning approach to predicting patterns in lateral inhibition systems. SIAM Journal on Applied Dynamical Systems, vol.12, no.4, pages 2012-2031, 2013.

\section{Conference Papers:}

[A14] Samuel Coogan and Murat Arcak. Dynamical properties of a compartmental model for traffic networks. In Proceedings of the 2014 American Control Conference, pages 2511-2516, Portland, Oregon, June 2014.

[A15] Yusef Shafi and Murat Arcak. An adaptive algorithm for synchronization in diffusively coupled systems. In Proceedings of the 2014 American Control Conference, pages 22202225, Portland, Oregon, June 2014.

[A16] Samuel Coogan and Murat Arcak. Freeway traffic control from linear temporal logic specifications. In Proceedings of the 5th IEEE/ACM International Conference on CyberPhysical Systems, pages 36-47, Berlin, Germany, April 2014.

[A17] Samuel Coogan and Murat Arcak. Verifying safety of interconnected passive systems using SOS programming. In Proceedings of the 52nd IEEE Conference on Decision and Control, pages 5951-5956, Florence, Italy, December 2013.

[A18] Yusef Shafi, Murat Arcak and Mihailo Jovanović. Synchronization of limit cycle oscillations in diffusively-coupled systems. In Proceedings of the 2013 American Control Conference, pages 4771-4776, Washington, DC, June 2013.

[A19] Ana Sofia Rufino Ferreira and Murat Arcak. Predicting patterns in lateral inhibition systems via graph partitioning. In Proceedings of the 2013 American Control Conference, pages 5497-5502, Washington, DC, June 2013.

[A20] Yusef Shafi, Zahra Aminzare, Murat Arcak and Eduardo Sontag. Spatial uniformity in diffusively-coupled systems using $L_{2}$ norm contractions. In Proceedings of the 2013 American Control Conference, pages 5529-5534, Washington, DC, June 2013.

[A21] Justin Hsia, William Holtz, Michel Maharbiz and Murat Arcak. New architecture for patterning gene expression using zinc finger proteins and small RNAs. In Proceedings 
of the 51st IEEE Conference on Decision and Control, pages 1633-1638, Maui, Hawaii, December 2012.

[A22] Samuel Coogan and Murat Arcak. Guard synthesis for safety of hybrid systems using sum of squares programming. In Proceedings of the 51st IEEE Conference on Decision and Control, pages 6138-6143, Maui, Hawaii, December 2012.

[A23] Murat Arcak. Synchronization and pattern formation in diffusively coupled systems. In Proceedings of the 51st IEEE Conference on Decision and Control, pages 7184-7192, Maui, Hawaii, December 2012.

[A24] Ana Sofia Rufino Ferreira, Murat Arcak and Eduardo Sontag. A decomposition-based approach to stability analysis of large-scale stochastic systems. In Proceedings of the 2012 American Control Conference, pages 6382-6387, Montréal, Canada, June 2012.

[A25] Erin Summers, Murat Arcak and Andrew Packard. Further results on delay robustness of interconnected passive systems. In Proceedings of the 2012 American Control Conference, pages 4697-4702, Montréal, Canada, June 2012.

[A26] Samuel Coogan and Murat Arcak. Formation control with size scaling using relative displacement feedback. In Proceedings of the 2012 American Control Conference, pages 3877-3882, Montréal, Canada, June 2012.

[A27] Murat Arcak. On biological pattern formation by contact inhibition. In Proceedings of the 2012 American Control Conference, pages 3439-3444, Montréal, Canada, June 2012.

[A28] Yusef Shafi, Murat Arcak, Laurent El Ghaoui. Graph weight design for Laplacian eigenvalue constraints with multi-agent systems applications. In Proceedings of the 50th IEEE Conference on Decision and Control and European Control Conference, pages 5541-5546, Orlando, Florida, December 2011.

[A29] Samuel Coogan, Murat Arcak, Magnus Egerstedt. Scaling the size of a multiagent formation via distributed feedback. In Proceedings of the 50th IEEE Conference on Decision and Control and European Control Conference, pages 994-999, Orlando, Florida, December 2011. 